University of Nebraska - Lincoln

DigitalCommons@University of Nebraska - Lincoln

\title{
Effect of spin-dependent screening on tunneling electroresistance and tunneling magnetoresistance in multiferroic tunnel junctions
}

\author{
M. Ye. Zhuravlev \\ University of Nebraska - Lincoln \\ S. Maekawa \\ Institute for Materials Research, Tohoku University, Sendai 980-8577, Japan \\ Evgeny Y. Tsymbal \\ University of Nebraska-Lincoln, tsymbal@unl.edu
}

Follow this and additional works at: https://digitalcommons.unl.edu/physicstsymbal

Part of the Condensed Matter Physics Commons

Zhuravlev, M. Ye.; Maekawa, S.; and Tsymbal, Evgeny Y., "Effect of spin-dependent screening on tunneling electroresistance and tunneling magnetoresistance in multiferroic tunnel junctions" (2010). Evgeny Tsymbal Publications. 34.

https://digitalcommons.unl.edu/physicstsymbal/34

This Article is brought to you for free and open access by the Research Papers in Physics and Astronomy at DigitalCommons@University of Nebraska - Lincoln. It has been accepted for inclusion in Evgeny Tsymbal Publications by an authorized administrator of DigitalCommons@University of Nebraska - Lincoln. 


\title{
Effect of spin-dependent screening on tunneling electroresistance and tunneling magnetoresistance in multiferroic tunnel junctions
}

\author{
M. Ye. Zhuravlev, ${ }^{1,2, *}$ S. Maekawa, ${ }^{3,4}$ and E. Y. Tsymbal ${ }^{1, \dagger}$ \\ ${ }^{1}$ Department of Physics and Astronomy, Nebraska Center for Materials and Nanoscience, University of Nebraska, Lincoln, \\ Nebraska 68588-0111, USA \\ ${ }^{2}$ Kurnakov Institute for General and Inorganic Chemistry, Russian Academy of Sciences, 119991 Moscow, Russia \\ ${ }^{3}$ Institute for Materials Research, Tohoku University, Sendai 980-8577, Japan \\ ${ }^{4}$ CREST, Japan Science and Technology Agency, Sanbancho, Tokyo 102-0075, Japan \\ (Received 17 January 2010; revised manuscript received 10 March 2010; published 25 March 2010)
}

\begin{abstract}
Using a ferroelectric barrier as a functional material in a (magnetic) tunnel junction has recently attracted significant interest due to new functionalities not available in conventional tunnel junctions. Switching a ferroelectric polarization of the barrier alters conductance resulting in a tunneling electroresistance (TER) effect. Using a ferroelectric barrier in a magnetic tunnel junction makes it mutiferroic where TER coexists with tunneling magnetoresistance (TMR). Here we develop a simple model for a multiferroic tunnel junction (MFTJ) which consists of two ferromagnetic electrodes separated by a ferroelectric barrier layer. The model explicitly includes the spin-dependent screening potential and thus extends previously developed models for FTJs and MFTJs. Our results demonstrate that the effect of spin-dependent screening may be sizable and may provide significant contributions to TMR and TER in MFTJs. We find that, similar to FTJs with a composite (ferroelectric/dielectric) barrier layer, the TER in a MFTJ with such a barrier is dramatically enhanced indicating that the resistance ratio between the states corresponding to the opposite polarization orientations may be as high as $10^{4}$ and even higher. Our results demonstrate the possibility of four resistance states in MFTJs with a pronounced difference in resistance and a possibility to control these resistance by an electric field (through ferroelectric polarization of the barrier) and by a magnetic field (through magnetization configuration of the electrodes). These functionalities may be interesting to device applications of MFTJs.
\end{abstract}

DOI: 10.1103/PhysRevB.81.104419 PACS number(s): 73.40.Gk, 77.55.-g, 72.25.-b, 73.40.Rw

\section{INTRODUCTION}

Ferroelectric tunnel junctions (FTJ) have recently aroused considerable interest due to interesting physics involved and potential applications as nanoscale resistive switching devices. ${ }^{1}$ A FTJ consists of two metal electrodes separated by a nm-thick ferroelectric barrier which allows electron tunneling through it. Recent experimental ${ }^{2-4}$ and theoretical ${ }^{5-8}$ studies of perovskite ferroelectric oxide thin films have demonstrated that ferroelectricity persists down to a nanometer scale, which makes it possible to use ferroelectrics as functional tunnel barriers in FTJs. Contrary to ferroelectric capacitors where leakage currents are detrimental to the device performance, ${ }^{9}$ the conductance of a FTJ is the functional characteristic of the device. The key property is tunneling electroresistance (TER) that is the change in resistance of a FTJ with reversal of ferroelectric polarization. Based on simple models it was predicted that TER in FTJs can be sizable due to the change in the tunneling potential barrier dependent on ferroelectric polarization orientation. ${ }^{10,11}$ These results were elaborated using first-principles calculations of transport properties of FTJs, showing, in addition, the importance of interface bonding and barrier decay rate effects on TER. ${ }^{12,13}$ Very recently three experimental groups have reported observations of the TER effect associated with the switching of ferroelectric (FE) polarization of $\mathrm{BaTiO}_{3}$ (Refs. 14 and 15) and $\mathrm{Pb}_{1-\mathrm{x}} \mathrm{Zr}_{\mathrm{x}} \mathrm{TiO}_{3}$ (Ref. 16) ferroelectric films. As predicted, ${ }^{10,17}$ the observed effects are really giant, showing the resistance change by two-three orders in magnitude. These experimental results proved the concept of FTJ and demonstrated the capability of thin-film ferroelectrics to serve as a nanoscale material that can act as a switch to store binary information.

Functionalities of a FTJ can be enhanced in a multifferoic tunnel junction (MFTJ) first introduced in Ref. 18. A MFTJ represents a FTJ with ferromagnetic electrodes or equivalently a magnetic tunnel junction (MTJ) with a ferroelectric barrier. ${ }^{1}$ Thus, the multiferroicity of such a junction follows from its composite nature involving two ferroic components-ferroelectric and ferromagnetic. Electron tunneling from a ferromagnetic metal electrode through a thin insulating barrier layer is spin polarized. ${ }^{19}$ As the consequence, in a MTJ the tunneling current depends on the relative magnetization orientation of the two FM electrodes, a phenomenon known as tunneling magnetoresistance (TMR) ${ }^{20}$ In a MFTJ the TER and TMR effects coexist, ${ }^{18}$ and therefore, a MFTJ represents a four-state resistance device where resistance can be switched both by electric and magnetic fields. ${ }^{13}$ Another type of MFTJ is feasible in which a single-phase multiferroic is used as barrier. ${ }^{21}$ In such a MFTJ the TMR effect is due to spin filtering properties of the mutiferroic barrier while the TER effect is due to change in the barrier potential profile when the polarization is switched. ${ }^{22}$

In both FTJs and MFTJs the important contribution to TER originates from the electrostatic effect resulting from the incomplete screening of polarization charges at the interfaces. ${ }^{10}$ This creates finite-size charge-depletion (accumulation) regions and hence an asymmetric potential profile in FTJs with different electrodes or/and with a composite barrier. ${ }^{17}$ If the electrodes are ferromagnetic the screening of 


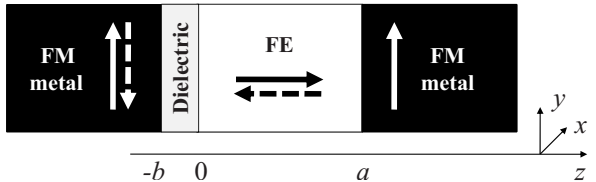

FIG. 1. Geometry of a MFTJ that consists of two FM metal electrodes separated by a FE barrier layer. A thin dielectric barrier may $(b \neq 0)$ or may not $(b=0)$ be inserted at the FM/FE interface. Conductance of the MFTJ is controlled by both the orientation of ferroelectric polarization of the barrier and by magnetization alignment of the two ferromagnetic electrodes, as indicated by arrows.

polarization charges is expected to be spin dependent $t^{23,24}$ due the exchange splitting of the spin bands in ferromagnetic metal electrodes. The spin-dependent screening is the origin of interface magnetoelectric effects, ${ }^{25-29}$ which complement interface magnetoelectric effects driven by strain ${ }^{30,31}$ and interface bonding ${ }^{32-35}$ (see Ref. 36 for a recent review of this and related fields of research).

The existing studies of the TER effect in MFTJs based on free-electron models have ignored the spin-dependent screening at the interfaces and its contribution to TMR..$^{18,22}$ Although such effects are captured in first-principles calculations, ${ }^{13}$ the development of a simple model could be useful for a deeper understanding of TER and its interplay with TMR in MFTJs. Here by employing a theory developed by Zhang ${ }^{23}$ we explicitly include the spin-dependent screening in a free-electron model for MFTJs, extending our previously developed model for FTJ with asymmetric electrodes ${ }^{10}$ and a composite (ferroelectric/dielectric) barrier layer ${ }^{17}$ to FTJs with ferromagnetic electrodes, i.e., MFTJs. This also generalizes our original model for MFTJs (Ref. 18) to include spin-dependent screening.

\section{SPIN-DEPENDENT SCREENING}

We consider a MFTJ which consists of two ferromagnetic metal electrodes separated by a composite barrier that includes a ferroelectric layer of thickness $a$ and a dielectric layer of thickness $b$, as shown in Fig. 1. Within a Stoner model $^{37}$ for itinerant magnetism each of the ferromagnetic electrodes can be described by two spin bands which are split due to the exchange coupling. The effective spin-dependent potential $V_{0}^{\sigma}$ in the bulk ferromagnet is given by

$$
e V_{0}^{\sigma}=e V_{0}+(-1)^{\sigma+1 / 2} \Delta / 2,
$$

where $\sigma= \pm 1 / 2$ (or equivalently $\sigma=\uparrow, \downarrow$ ) is the spin index and $\Delta$ is the exchange splitting. The latter is determined by the Stoner exchange parameter $J$ and the equilibrium spin density $m$ so that

$$
\Delta=J m,
$$

where the spin density is defined by

$$
m=n^{\uparrow}-n^{\downarrow}
$$

and $e n^{\sigma}$ is the spin-dependent charge density in the bulk electrodes. A spontaneous polarization in the ferroelectric barrier produces the interface polarization charge which is screened in the metal electrodes. This screening is spin dependent and consequently the induced charge density has two nonequal spin contributions $\delta n^{\uparrow}(z)$ and $\delta n^{\downarrow}(z)$, which depend on coordinate $z$ perpendicular to the planes

$$
\delta n(z)=\delta n^{\uparrow}(z)+\delta n^{\downarrow}(z) .
$$

This leads to the induced nonuniform spin density near the interface

$$
\delta m(z)=\delta n^{\uparrow}(z)-\delta n^{\downarrow}(z) .
$$

The induced charge and spin densities produce two additional contributions to the spin-dependent potential $\delta V^{\sigma}(z)$ (Ref. 23)

$$
e \delta V^{\sigma}(z)=e V_{c}(z)+(-1)^{\sigma+1 / 2} J \delta m(z) .
$$

The first term is associated with the Coulomb potential $V_{c}(z)$ which is produced by the induced change density $\delta n(z)$. This potential satisfies the Poisson's equation

$$
\frac{d^{2} V_{c}(z)}{d x^{2}}=-\frac{e}{\varepsilon_{0}} \delta n(z),
$$

where the dielectric permittivity of the electrodes is assumed to be equal to the dielectric permittivity of vacuum $\varepsilon_{0}$. The second term in Eq. (6) is the effective exchange energy associated with the induced spin density. This term is analogous to the last term in Eq. (1) but does not have a factor of $\frac{1}{2}$ which appears in Eq. (1) due to the double counting. We obtain the induced charge density in the electrodes using the Thomas-Fermi approximation

$$
\delta n^{\sigma}(z)=-e \rho^{\sigma} \delta V^{\sigma}(z),
$$

where $\left.\rho^{\sigma} \equiv \frac{d n^{\sigma}(E)}{d E}\right|_{E=E_{F}}$ is the spin-dependent density of states (DOS) at the Fermi energy $E_{F}$. Substituting Eq. (6) in Eq. (8) and solving with respect to $\delta n^{\uparrow}$ and $\delta n^{\downarrow}$ we find

$$
\delta n^{\sigma}(z)=-\frac{\left[1+2 J \rho^{-\sigma}\right] \rho^{\sigma}}{1+J \rho} e V_{c}(z),
$$

where $\rho=\rho^{\downarrow}+\rho^{\uparrow}$ is the total DOS at the Fermi energy. The induced spin-dependent potential given by Eq. (6) can therefore be written as follows:

$$
e \delta V^{\sigma}(z)=e V_{c}(z)\left[1-\frac{J\left(\rho^{\sigma}-\rho^{-\sigma}\right)}{1+J \rho}\right] .
$$

Equations (9) and (7) lead to the induced Coulomb potential given by

$$
\frac{d^{2} V_{c}(z)}{d z^{2}}=\frac{V_{c}(z)}{\lambda^{2}},
$$

where the screening $\lambda$ length is defined as follows:

$$
\lambda=\left(\frac{e^{2}}{\varepsilon_{0}} \frac{\rho+4 J \rho^{\downarrow} \rho^{\uparrow}}{1+J \rho}\right)^{-1 / 2} .
$$

To obtain the explicit form of potential $\delta V_{c}(z)$ we need to solve Eq. (11) using appropriate boundary conditions. Assuming the short-circuit boundary conditions for the bulk electrodes leads to the solution 


$$
\delta V_{c}(z)=\left\{\begin{array}{cc}
V_{c}^{L} e^{(z+b) / \lambda_{L}} & z<-b \\
V_{c}^{R} e^{-(z-a) / \lambda_{R}} & z>a .
\end{array}\right.
$$

Here $\lambda_{L}\left(\lambda_{R}\right)$ is the screening length in the left (right) electrodes and $V_{c}^{L}\left(V_{c}^{R}\right)$ is the amplitude of the Coulomb potential at the left (right) interface between the ferromagnet and the barrier. The amplitudes of the Coulomb potential at the interfaces are determined by the electrostatic boundary conditions

$$
\left\{\begin{array}{l}
\frac{V_{c}^{0}-V_{c}^{R}}{a}=\frac{P-\sigma}{\varepsilon_{0} \varepsilon_{f}} \\
\frac{V_{c}^{L}-V_{c}^{0}}{b}=\frac{-\sigma}{\varepsilon_{0} \varepsilon_{d}},
\end{array}\right.
$$

where $P$ is the spontaneous polarization of the ferroelectric layer, $\varepsilon_{f}$ and $\varepsilon_{d}$ are the dielectric constants (relative dielectric permittivities) of the ferroelectric and dielectric layers, respectively, and $V_{c}^{0}$ is the electrostatic potential at $z=0$. Due to the charge conservation the induced surface charge density $\sigma$ is the same for both electrodes and can be found from

$$
\sigma=\int_{-\infty}^{-b} \varepsilon_{0}\left[\frac{\partial^{2} V_{c}(z)}{\partial z^{2}}\right] d z=-\int_{a}^{\infty} \varepsilon_{0}\left[\frac{\partial^{2} V_{c}(z)}{\partial z^{2}}\right] d z
$$

so that

$$
V_{c}^{L, R}= \pm \frac{\sigma \lambda_{L, R}}{\varepsilon_{0}}
$$

Using Eqs. (14) and (16) we find

$$
\begin{gathered}
\sigma=\frac{a \varepsilon_{d} P}{a \varepsilon_{d}+b \varepsilon_{f}+\varepsilon_{f} \varepsilon_{d}\left(\lambda_{L}+\lambda_{R}\right)}, \\
V_{c}^{0}=\frac{1}{\varepsilon_{0}} \frac{\left(a \lambda_{L} \varepsilon_{d}+a b\right) P}{a \varepsilon_{d}+b \varepsilon_{f}+\varepsilon_{f} \varepsilon_{d}\left(\lambda_{L}+\lambda_{R}\right)}, \\
V_{c}^{L, R}= \pm \frac{1}{\varepsilon_{0}} \frac{a \lambda_{L, R} \varepsilon_{d} P}{a \varepsilon_{d}+b \varepsilon_{f}+\varepsilon_{f} \varepsilon_{d}\left(\lambda_{L}+\lambda_{R}\right)} .
\end{gathered}
$$

Corresponding values of the charge and the potential for a MFTJ system with no dielectric layer can be easily found from Eqs. (17) by assuming $b=0$.

Within a free-electron model we have

$$
\begin{gathered}
n^{\uparrow, \downarrow}=\frac{1}{6 \pi^{2}}\left[\frac{2 m_{e}}{\hbar^{2}}\left(E_{F} \pm \Delta / 2\right)\right]^{3 / 2}, \\
\rho^{\uparrow, \downarrow}\left(E_{F}\right)=\frac{3}{2} \frac{n^{\uparrow, \downarrow}}{E_{F} \pm \Delta / 2},
\end{gathered}
$$

where $E_{F}$ is the Fermi energy (measured with respect to energy $\left.e V_{0}\right), m_{e}$ is an effective mass and all the quantities entering Eqs. (18) and (19) may be different for the two electrodes.

Figure 2(a) shows the screening length in a ferromagnetic electrode as a function of exchange splitting of spin bands $\Delta$. In the calculations we assumed a free-electron mass for $m_{e}$ and the Fermi energy $E_{F}=2 \mathrm{eV}$ so that the exchange split-

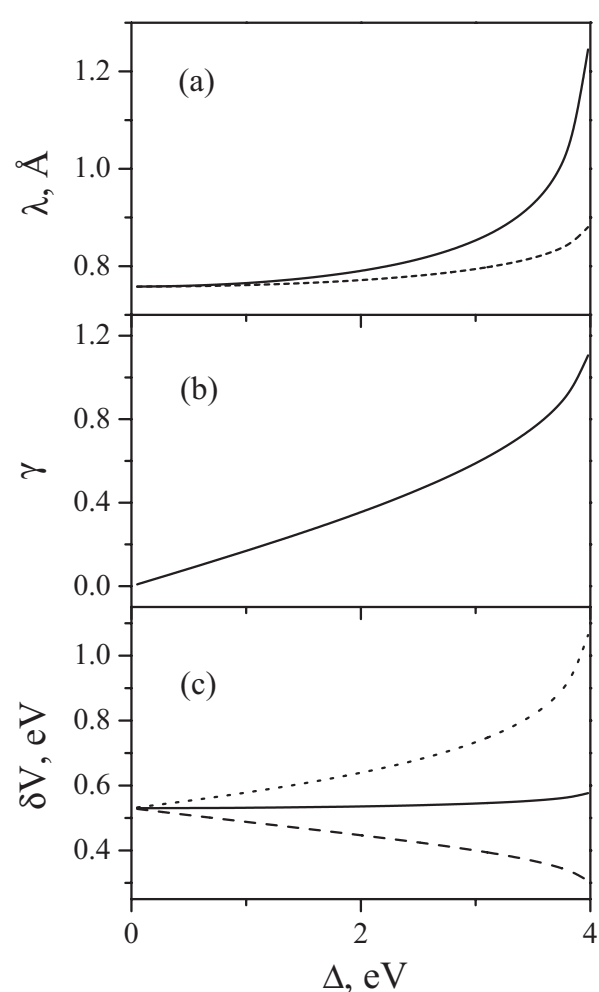

FIG. 2. Spin-dependent screening effects as a function exchange splitting $\Delta$ in the ferromagnetic electrode. (a) Screening length for $J \neq 0$ (solid line) and for $J=0$ (dashed line). (b) Spin-dependent contribution to the potential from Eq. (10): $\gamma=2 J\left(\rho^{\uparrow}-\rho^{\downarrow}\right) /(1+J \rho)$. (c) Magnitude of the screening potential at the FM/FE interface $(z=0)$ in a FM/FE/NM junction without the spin-dependent contribution (solid line) and with the spin-dependent contribution for minority-spin (dotted line) and majority-spin (dashed line) electrons for $\varepsilon_{f}=90$ and $E_{F}=2 \mathrm{eV}$.

ting varies from $\Delta=0$ (no spin splitting) to $\Delta=4 \mathrm{eV}$ (half metal). It is seen that ignoring the spin-dependent contribution (i.e., assuming that the screening length depends only on the total density of states) leads to sizable deviation from a more accurate model where the spin-dependent contribution is taken into account. The importance of the spin-dependent contribution is also evident from Fig. 2(b) which shows the screening-induced spin splitting of the potential at the interface that enters Eq. (10), i.e., $\gamma=2 J\left(\rho^{\uparrow}-\rho^{\downarrow}\right) /(1+J \rho)$. It is seen that the values are sizable (compared to unity) even for a moderate exchange splitting and exceed unity when the exchange splitting approaches $4 \mathrm{eV}$.

These effects are expected to have a notable contribution to spin-dependent tunneling in MTJs (Ref. 23) including those with a ferroelectric barrier. This fact is seen from Fig. 2(c) which shows the screening potential at the ferromagnet $(\mathrm{FM}) / \mathrm{FE}$ interface, $\delta V^{\sigma}(z=0)$, in a $\mathrm{FM} / \mathrm{FE} /$ normal metal (NM) junction with and without the spin-dependent contribution as a function of the exchange splitting in the ferromagnet. Here we assume that the saturation polarization of a ferroelectric is $P=40 \mu \mathrm{C} / \mathrm{cm}^{2}$ and the dielectric constant of a ferroelectric in the saturation state is $\varepsilon_{f}=90$. A sizable difference is seen, especially for large values of $\Delta$, indicating the importance of spin-dependent splitting for tunneling conductance. 


\section{TUNNELING CONDUCTANCE}

The tunneling conductance per spin channel $\sigma=\uparrow, \downarrow$ per unit area is calculated using the Landauer formula

$$
G^{\sigma}=\frac{e^{2}}{h} \int \frac{d^{2} \mathbf{k}_{\|}}{(2 \pi)^{2}} T^{\sigma}\left(E_{F}, \mathbf{k}_{\|}\right),
$$

where $T\left(E_{F}, \mathbf{k}_{\|}\right)$is the transmission coefficient at the Fermi energy $E_{F}$ for a given value of the transverse wave vector $\mathbf{k}_{\|}$. The transmission coefficient is obtained from the solution of the Schrödinger equation for an electron moving in a potential $V(z)$ by imposing a boundary condition of the incoming plane wave normalized to a unit flux density and by calculating the amplitude of the transmitted plane wave. The solution is obtained numerically for the potential $V(z)$ which is the superposition of the electrostatic potential, the exchange potential, and the stepwise potential originating from the variation in the conduction-band minima across the junction. For a given Fermi energy $E_{F}$ in the metal electrodes, the latter determines the barrier heights $U_{d}$ and $U_{f}$ for the nonpolar dielectric and ferroelectric layers, respectively. We assume that electrons have a free-electron mass, the Fermi energy is $E_{F}=2 \mathrm{eV}$, and the ferroelectric barrier height (with respect to $E_{F}$ ) is $U_{f}=0.6 \mathrm{eV}$. We define the TER ratio as follows: TER $=G_{L} / G_{R}$, where $G_{L}$ and $G_{R}$ are conductances of a MFTJ for polarization in the barrier pointing left $\left(P_{L}\right)$ and right $\left(P_{R}\right)$, respectively (see Fig. 1). The TMR ratio is defined as TMR $=\left(G_{A P}-G_{P}\right) /\left(G_{A P}+G_{P}\right)$, where $G_{P}$ are $G_{A P}$ conductances for parallel $\left(M_{P}\right)$ and antiparallel $\left(M_{A P}\right)$ magnetization of the FM electrodes.

First, we apply the theory presented to calculate the spindependent conductance for a FM/FE/NM junction considered in the preceding section [Fig. 2(c)] to illustrate the significance of the spin-dependent contribution to the potential. The results are displayed in Fig. 3(a), which shows the total conductance calculated with and without spin-dependent contribution to the screening. In accordance to Fig. 2 we see a sizable difference between the two indicating the significance of the spin-dependent contribution.

As expected, switching of ferroelectric polarization leads to the TER effect. As seen from Fig. 3(b), the magnitude of this effect increases with the exchange splitting of spin bands in the ferromagnetic electrode which is the consequence of the increasing asymmetry between the left and right leads. While the screening length of the right electrode remains constant, the screening length of left electrode is increasing with $\Delta$ [see Fig. 2(a)] which leads to the enhancement of TER at large $\Delta$ [see Fig. 3(b)]. This result is consistent with that of Ref. 10 even though the details of the electronic structure are more complex in the present case due to spin-split bands of the left ferromagnetic electrode.

As was demonstrated previously, ${ }^{18}$ the spin polarization of tunneling conductance from a ferromagnetic metal through a ferroelectric barrier depends on ferroelectric polarization orientation. This is also the case for a tunnel junction considered here. We define the spin polarization of conductance in the standard way, ${ }^{19}$ i.e., $\left(G_{m a j}-G_{m i n}\right) /\left(G_{m a j}+G_{m i n}\right)$, where $G_{m a j}\left(G_{\text {min }}\right)$ in the conductance for majority (minority) spin electrons. As is evident from Fig. 3(c), the spin polar-

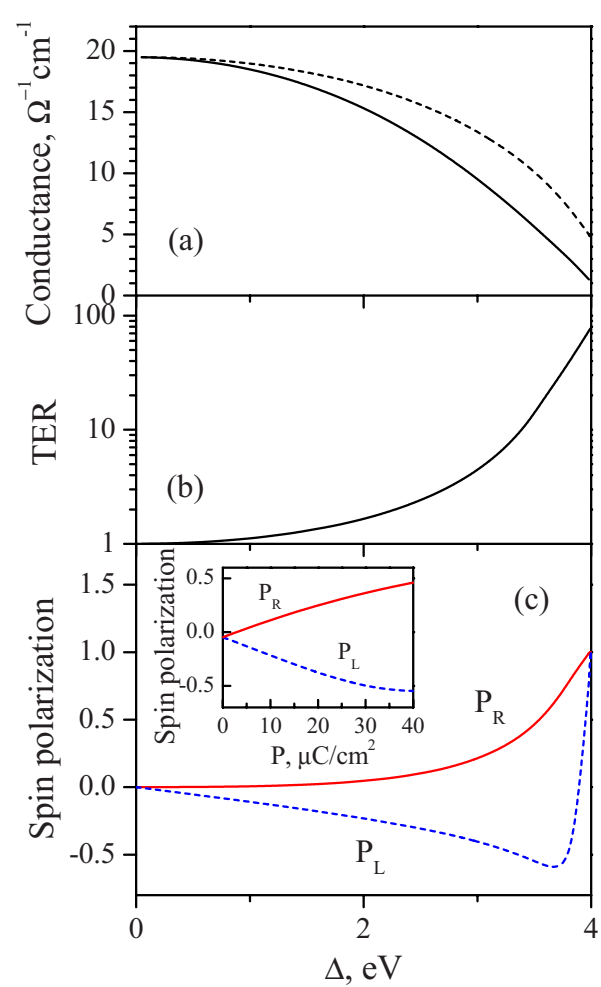

FIG. 3. (Color online) (a) Conductance for ferroelectric polarization pointing right with (solid line) and without (dashed line) spin-dependent contribution to the screening potential, (b) TER, and (c) spin polarization of the tunneling conductance for ferroelectric polarization pointing right (solid line) and left (dashed line) in a FM/FE/NM tunnel junction as functions of exchange splitting in the ferromagnetic electrode for $a=2.5 \mathrm{~nm}, P=40 \mu \mathrm{C} / \mathrm{cm}^{2}, \varepsilon_{f}=90$ $U_{f}=0.6 \mathrm{eV}$, and $E_{F}=2 \mathrm{eV}$. The inset shows the transport spin polarization as a function of ferroelectric polarization in the barrier for $\Delta=3.5 \mathrm{eV}$.

ization changes significantly when the ferroelectric polarization alters its direction from pointing left to right. Notable that for broad range of values $\Delta$ the spin polarization has different sign for the two opposite orientations of ferroelectric polarization. This is the consequence of a different potential barrier height at the FM/FE interface for opposite polarizations. According to the free-electron model for tunneling across a rectangular barrier, ${ }^{38}$ the spin polarization in the asymptotic limit of a thick barrier is determined by

$$
P_{s p}=\frac{\kappa^{2}-k^{\downarrow} k^{\uparrow}}{\kappa^{2}+k^{\downarrow} k^{\uparrow}} \frac{k^{\uparrow}-k^{\downarrow}}{k^{\uparrow}+k^{\downarrow}},
$$

where $k^{\downarrow}\left(k^{\uparrow}\right)$ is the Fermi wave vector in the ferromagnetic electrode and $\kappa$ is the decay constant in the barrier. With increasing the exchange splitting in Fig. 3(c) the absolute value of the spin polarization $P_{s p}$ increases due to the second term in Eq. (21) which is determined solely by the electrode. A different sign for opposite ferroelectric polarization orientations comes from the first term in Eq. (21) which is controlled by the interface transmission function. ${ }^{39}$ For ferroelectric polarization pointing right the effective barrier height and thus value of $\kappa$ at the interface is relatively high making the $P_{s p}$ positive ${ }^{40}$ whereas for ferroelectric polarization 
pointing left the effective barrier and thus value of $\kappa$ at the interface are low making the $P_{s p}$ polarization negative. For large $\Delta$, however, the spin polarization becomes positive (due to $k^{\downarrow} k^{\uparrow}$ tending to zero) independent of the ferroelectric state. For $\Delta=4 \mathrm{eV}$ the tunneling current is fully spin polarized, as expected for a half-metallic injector.

The sensitivity of the transport spin polarization to the potential change at the interface makes it dependent on the magnitude of polarization of the ferroelectric barrier, $P$. This is seen from the inset of Fig. 3(c), where the spin polarization is calculated as a function of $P$ for $\Delta=3.5 \mathrm{eV}$ at which the spin polarization has opposite sign for the $P_{L}$ and $P_{R}$ states. With increasing $P$ the asymmetry is increasing in response to the gradual change in the potential step at the interface.

\section{TER AND TMR EFFECTS IN MFTJS}

Using a ferroelectric barrier in a MTJ makes it multiferroic where four resistance states are allowed corresponding to two magnetization alignments of ferromagnetic electrodes, i.e., parallel and antiparallel $\left(M_{P}\right.$ and $M_{A P}$ states, respectively) and two orientations of ferroelectric polarization, i.e., pointing left and right $\left(P_{L}\right.$ and $P_{R}$ states, respectively). To illustrate this behavior we consider a model FM/FE/FM junction where left electrode is a half metal, i.e., $\Delta_{L}$ $=4.0 \mathrm{eV}$ and the exchange splitting in right electrode is assumed to be $\Delta_{R}=3.5 \mathrm{eV}$. Figure 4 shows results of calculations as a function of the magnitude of polarization $P$ in the barrier. As seen from Fig. 4(a), for $P=0$ there are two resistance states corresponding to a small conductance difference for the $M_{P}$ and $M_{A P}$ states [see also Fig. 4(b) for $P=0$ ]. For nonzero polarization, however, four resistance states develop with a large difference in conductance that increases with $P$.

The presence of the four resistance states implies that TMR can be controlled by ferroelectric polarization orientation [Fig. 4(b)] and TER can be controlled by magnetization alignment of the electrodes [Fig. 4(c)]. As seen from Fig. 4(b), for a nonzero ferroelectric polarization $P$ in the barrier, TMR values have different sign for the $P_{L}$ and $P_{R}$ states. This behavior is reminiscent to that seen in the transport spin polarization for a FE/FE/NM junction [inset of Fig. 3(c)]. Since the TMR can be considered as a product of the two transmission functions at the right and left interfaces, ${ }^{39}$ it is expected that TMR should have similar behavior but opposite sign to that seen for the spin polarization in the inset of Fig. 3(c). This is due to the fact that the left electrode in the MFTJ is assumed to be fully spin polarized $\left(\Delta_{L}=4.0 \mathrm{eV}\right)$ and the orientation of ferroelectric polarization with respect to the right electrode $\left(\Delta_{R}=3.5 \mathrm{eV}\right)$ is reversed as compared to that in the inset of Fig. 3(c) with respect the left electrode. With the increasing ferroelectric polarization $P$ in the barrier, asymmetry in TMR values increases reproducing a similar behavior of the transport spin polarization. Like the respective spin polarization [Fig. 3(c)], the TMR magnitude strongly depends on the exchange splitting in the ferromagnetic electrode [see the inset of Fig. 4(b)].

Figure 4(c) shows that TER strongly depends on the magnetization alignment of the electrodes. As expected, ${ }^{10}$ with

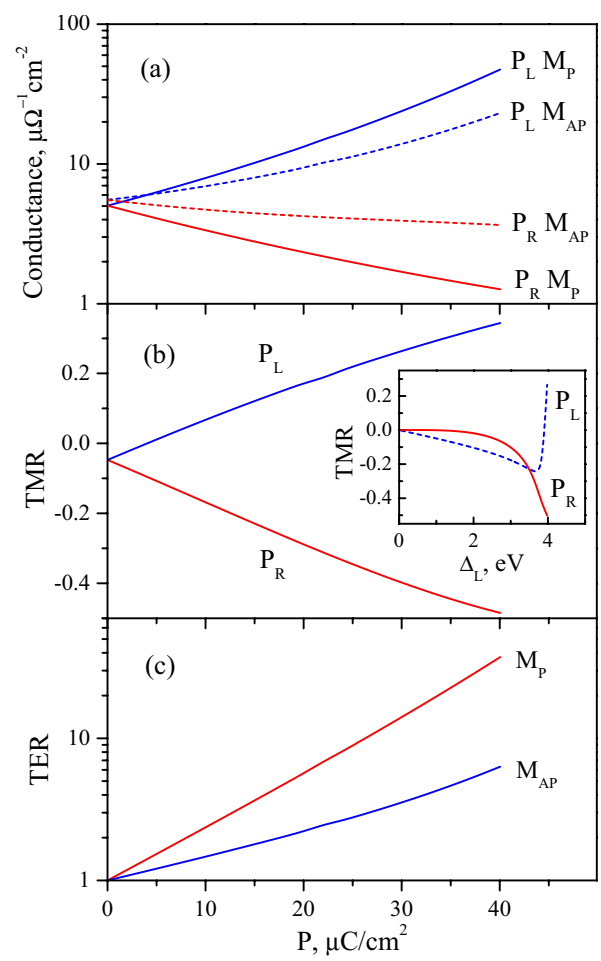

FIG. 4. (Color online) (a) Conductance (per unit area) for four resistance states, (b) TMR for two ferroelectric polarization orientations, and (c) TER for parallel and antiparallel magnetization of the electrodes in a FM/FE/FM MFTJ as a function of ferroelectric polarization $P$ in a barrier. $a=2.5 \mathrm{~nm}, P=40 \mu \mathrm{C} / \mathrm{cm}^{2}, U_{f}$ $=0.6 \mathrm{eV}, \Delta_{L}=4.0 \mathrm{eV}, \Delta_{R}=3.5 \mathrm{eV}, E_{F}=2 \mathrm{eV}$, and $\varepsilon_{f}=90$. The inset shows TMR as a function of exchange splitting $\Delta_{L}$ for two polarization orientations.

increasing $P$ the TER grows significantly due to the increasing asymmetry in the potential profile for opposite orientations of ferroelectric polarization. For a MFTJ this enhancement in TER is accompanied by a substantial difference in TER for parallel and antiparallel magnetizations. This effect is largely controlled by the spin-dependent potential at the interface. Results of the calculation which does not include the spin-dependant contribution to the potential (not shown) reveal significantly reduced values of TER and diminished asymmetry between TER values for the $M_{P}$ and $M_{A P}$ states.

Earlier we have demonstrated ${ }^{17}$ that using a layered composite barrier combining a functional ferroelectric film and a thin film of a nonpolar dielectric material considerably enhances TER. The effect occurs due to the change in the electrostatic potential (induced by polarization reversal) in the nonpolar film adjacent to one of the interfaces that acts as a switch changing its barrier height from a low-to-high value resulting in a dramatic change in the transmission across the FTJ. The same effect occurs in a MFTJ with an additional dielectric barrier layer inserted at the FM/FE interface (see Fig. 1). Figure 5(a) displays the dependence of TER as the function of the dielectric layer thickness $b$. As expected, the TER grows exponentially with $b$, indicating that the conductance ratio between the two polarization states in such MFTJs may reach $10^{4}$ and even higher.

A MFTJ adds an additional degree of freedom, as compared to a FTJ, that allows altering TER by changing the 


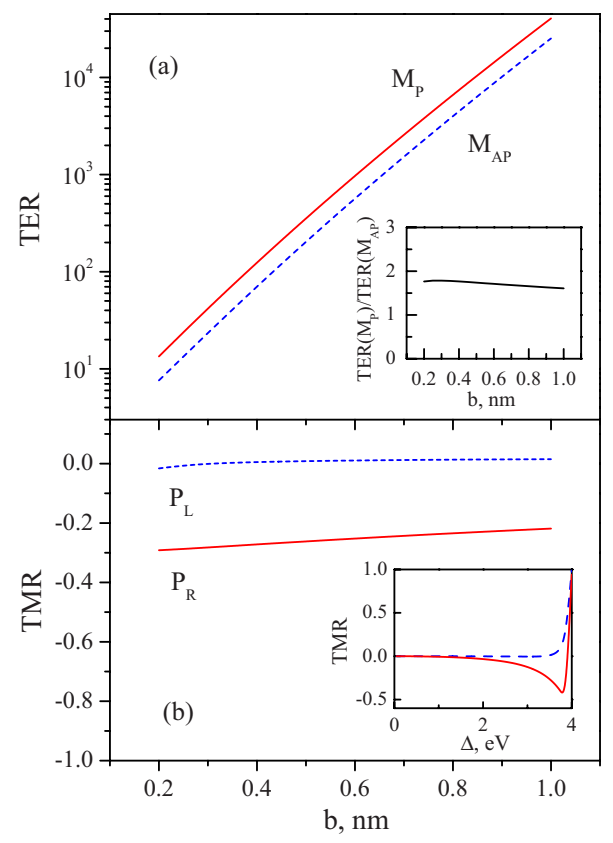

FIG. 5. (Color online) (a) TER for parallel and antiparallel magnetizations $\left(M_{P}\right.$ and $M_{A P}$, respectively) and (b) TMR for ferroelectric polarization pointing left and right $\left(P_{L}\right.$ and $P_{R}$, respectively) in a MFTJ with a dielectric layer as a function of the dielectric film thickness $b$ for $\Delta_{L}=\Delta_{R}=3.5 \mathrm{eV}, a=2.5 \mathrm{~nm}, P=40 \mu \mathrm{C} / \mathrm{cm}^{2}, U_{f}$ $=0.6 \mathrm{eV}, U_{b}=2.5 \mathrm{eV}, E_{F}=2 \mathrm{eV}$, and $\varepsilon_{f}=90, \varepsilon_{b}=10$. The inset of (a) shows a ratio of the TER values for the $M_{P}$ and $M_{A P}$ states. The inset of (b) shows TMR as a function of the exchange splitting in ferromagnetic electrodes, $\Delta=\Delta_{L}=\Delta_{R}$, for the $P_{L}$ and $P_{R}$ states and $b=0.5 \mathrm{~nm}$.

magnetization configuration of the electrodes. Figure 5(a) shows that the TER values differ for parallel and antiparallel magnetizations. The inset of Fig. 5(a) indicates that the ratio of the TER values for the $M_{P}$ and $M_{A P}$ states is about 1.7 and stable with respect to the dielectric layer thickness.

Multifunctionality of a MFTJ with a composite barrier is also manifested by a possibility to change TMR with ferroelectric polarization orientation in the barrier. As seen from Fig. 5(b), for chosen parameters the TMR is predicted to change from a relatively small value corresponding to the $P_{L}$ state to a sizable negative value corresponding to the $P_{R}$ state. This change is weakly dependent on the dielectric barrier thickness $b$. The inset of Fig. 5(b) indicates that TMR as a function of exchange splitting of the ferromagnetic electrodes, $\Delta=\Delta_{L}=\Delta_{R}$, behaves approximately as the square of the spin polarization of the tunneling current shown in Fig. 3(c), as expected for a MTJ (e.g., Ref. 41).

\section{CONCLUSIONS}

We have developed a simple model for a multiferroic tunnel junction which consists of two ferromagnetic electrodes separated by a ferroelectric barrier layer. The model explicitly includes the spin-dependent screening potential ${ }^{23}$ and thus extends our previously developed model for a FTJ with asymmetric electrodes ${ }^{10}$ to MFTJs. This also generalizes our original model for MFTJs (Ref. 18) to include the spindependent screening. Although the spin-dependent screening effects have been captured in first-principles calculations (see, e.g., Ref. 13), the availability of a simple model could be useful for a deeper understanding of TER and its interplay with TMR in MFTJs. Our results demonstrate that the effect of spin-dependent screening may be sizable and may provide significant contributions to TMR and TER in MFTJs. Similar to FTJs with a composite (ferroelectric/dielectric) barrier layer, ${ }^{17}$ the TER in a MFTJ with such a barrier is dramatically enhanced indicating that the resistance ratio between the states corresponding to the opposite polarization orientations may be as high as $10^{4}$ and even higher. Our results demonstrate the possibility of four resistance states in MFTJs with a pronounced difference in resistance and a possibility to control these resistances by an electric field (through ferroelectric polarization of the barrier) and by a magnetic field (through magnetization configuration of the electrodes). These functionalities may be interesting to device applications of MFTJs.

\section{ACKNOWLEDGMENTS}

M.Y.Z. is thankful to the Materials Research Science and Engineering Center (MRSEC) at the University of Nebraska for hospitality during his visit to Lincoln in summer 2009. This work was supported by NSF MRSEC (Grant No. 0820521), the Nanoelectronics Research Initiative, and the Nebraska Research Initiative.

\footnotetext{
*myezhur@gmail.com

†tsymbal@unl.edu

${ }^{1}$ E. Y. Tsymbal and H. Kohlstedt, Science 313, 181 (2006).

${ }^{2}$ D. D. Fong, G. B. Stephenson, S. K. Streiffer, J. A. Eastman, O. Auciello, P. H. Fuoss, and C. Thompson, Science 304, 1650 (2004)

${ }^{3}$ C. Lichtensteiger, J. M. Triscone, J. Junquera, and Ph. Ghosez, Phys. Rev. Lett. 94, 047603 (2005).

${ }^{4}$ D. A. Tenne, A. Bruchhausen, N. D. Lanzillotti-Kimura, A. Fainstein, R. S. Katiyar, A. Cantarero, A. Soukiassian, V. Vaithyanathan, J. H. Haeni, W. Tian, D. G. Schlom, K. J. Choi, D. M.
}

Kim, C. B. Eom, H. P. Sun, X. Q. Pan, Y. L. Li, L. Q. Chen, Q. X. Jia, S. M. Nakhmanson, K. M. Rabe, and X. X. Xi, Science 313, 1614 (2006).

${ }^{5}$ J. Junquera and Ph. Ghosez, Nature (London) 422, 506 (2003).

${ }^{6}$ N. Sai, A. M. Kolpak, and A. M. Rappe, Phys. Rev. B 72, 020101(R) (2005).

${ }^{7}$ C.-G. Duan, R. F. Sabirianov, W.-N. Mei, S. S. Jaswal, and E. Y. Tsymbal, Nano Lett. 6, 483 (2006).

${ }^{8}$ G. Gerra, A. K. Tagantsev, N. Setter, and K. Parlinski, Phys. Rev. Lett. 96, 107603 (2006).

${ }^{9}$ T. P. Ma and J. P. Han, IEEE Electron Device Lett. 23, 386 
(2002).

${ }^{10}$ M. Y. Zhuravlev, R. F. Sabirianov, S. S. Jaswal, and E. Y. Tsymbal, Phys. Rev. Lett. 94, 246802 (2005); 102, 169901 (2009).

${ }^{11}$ H. Kohlstedt, N. A. Pertsev, J. Rodríguez Contreras, and R. Waser, Phys. Rev. B 72, 125341 (2005).

${ }^{12}$ J. P. Velev, C.-G. Duan, K. D. Belashchenko, S. S. Jaswal, and E. Y. Tsymbal, Phys. Rev. Lett. 98, 137201 (2007).

13 J. P. Velev, C.-G. Duan, J. D. Burton, A. Smogunov, M. K. Niranjan, E. Tosatti, S. S. Jaswal, and E. Y. Tsymbal, Nano Lett. 9, 427 (2009).

${ }^{14}$ V. Garcia, S. Fusil, K. Bouzehouane, S. Enouz-Vedrenne, N. D. Mathur, A. Barthélémy, and M. Bibes, Nature (London) 460, 81 (2009).

${ }^{15}$ P. Maksymovych, S. Jesse, P. Yu, R. Ramesh, A. P. Baddorf, and S. V. Kalinin, Science 324, 1421 (2009).

${ }^{16}$ A. Gruverman, D. Wu, H. Lu, Y. Wang, H. W. Jang, C. M. Folkman, M. Y. Zhuravlev, D. Felker, M. Rzchowski, C.-B. Eom, and E. Y. Tsymbal, Nano Lett. 9, 3539 (2009).

${ }^{17}$ M. Y. Zhuravlev, Y. Wang, S. Maekawa, and E. Y. Tsymbal, Appl. Phys. Lett. 95, 052902 (2009).

${ }^{18}$ M. Y. Zhuravlev, S. S. Jaswal, E. Y. Tsymbal, and R. F. Sabirianov, Appl. Phys. Lett. 87, 222114 (2005).

${ }^{19}$ R. Meservey and P. M. Tedrow, Phys. Rep. 238, 173 (1994).

${ }^{20}$ E. Y. Tsymbal, O. N. Mryasov, and P. R. LeClair, J. Phys.: Condens. Matter 15, R109 (2003).

${ }^{21}$ M. Gajek, M. Bibes, S. Fusil, K. Bouzehouane, J. Fontcuberta, A. Barthélémy, and A. Fert, Nature Mater. 6, 296 (2007)

${ }^{22}$ S. Ju, T.-Y. Cai, G.-Y. Guo, and Z.-Y. Li, Phys. Rev. B 75, 064419 (2007).

${ }^{23}$ S. Zhang, Phys. Rev. Lett. 83, 640 (1999).

${ }^{24}$ C.-G. Duan, J. P. Velev, R. F. Sabirianov, Z. Zhu, J. Chu, S. S. Jaswal, and E. Y. Tsymbal, Phys. Rev. Lett. 101, 137201 (2008).

${ }^{25}$ J. M. Rondinelli, M. Stengel, and N. Spaldin, Nat. Nanotechnol. 3, 46 (2008).

${ }^{26}$ M. K. Niranjan, J. D. Burton, J. P. Velev, S. S. Jaswal, and E. Y.
Tsymbal, Appl. Phys. Lett. 95, 052501 (2009).

${ }^{27}$ H. Molegraaf, J. Hoffman, C. Vaz, S. Gariglio, D. van der Marel, C. Ahn, and J.-M. Triscone, Adv. Mater. 21, 3470 (2009).

${ }^{28}$ J. D. Burton and E. Y. Tsymbal, Phys. Rev. B 80, 174406 (2009).

${ }^{29}$ T. Cai, S. Ju, J. Lee, N. Sai, A. A. Demkov, Q. Niu, Z. Li, J. Shi, and E. Wang, Phys. Rev. B 80, 140415(R) (2009).

${ }^{30}$ W. Eerenstein, M. Wiora, J. L. Prieto, J. F. Scott, and N. D. Mathur, Nature Mater. 6, 348 (2007).

${ }^{31}$ S. Sahoo, S. Polisetty, C.-G. Duan, S. S. Jaswal, E. Y. Tsymbal, and C. Binek, Phys. Rev. B 76, 092108 (2007).

${ }^{32}$ C.-G. Duan, S. S. Jaswal, and E. Y. Tsymbal, Phys. Rev. Lett. 97, 047201 (2006).

${ }^{33}$ K. Yamauchi, B. Sanyal, and S. Picozzi, Appl. Phys. Lett. 91, 062506 (2007)

${ }^{34}$ M. Fechner, I. V. Maznichenko, S. Ostanin, A. Ernst, J. Henk, P. Bruno, and I. Mertig, Phys. Rev. B 78, 212406 (2008).

${ }^{35}$ M. K. Niranjan, J. P. Velev, C.-G. Duan, S. S. Jaswal, and E. Y. Tsymbal, Phys. Rev. B 78, 104405 (2008).

${ }^{36}$ K. F. Wang, J.-M. Liu, and Z. F. Ren, Adv. Phys. 58, 321 (2009).

${ }^{37}$ E. C. Stoner, Proc. R. Soc. London 165, 372 (1938).

${ }^{38}$ J. C. Slonczewski, Phys. Rev. B 39, 6995 (1989).

${ }^{39}$ K. D. Belashchenko, E. Y. Tsymbal, M. van Schilfgaarde, D. A. Stewart, I. I. Oleinik, and S. S. Jaswal, Phys. Rev. B 69, 174408 (2004).

${ }^{40}$ We note that Eq. (22) is derived for the asymptotic limit of a thick barrier where only $k_{\|}=0$ contribute to the conductance. In our case of a relatively thin barrier $a=2.5 \mathrm{~nm}$ and low-potential barrier height $U_{f}=0.6 \mathrm{eV}$ electrons with $k_{\|} \neq 0$ contribute to the conductance. For these electrons the effective decay constant $\kappa$ is such that $\kappa^{2}\left(\mathbf{k}_{\|}\right)>k^{\downarrow}\left(\mathbf{k}_{\|}\right) k^{\uparrow}\left(\mathbf{k}_{\|}\right)$making the integrated tunneling conductance positively spin polarized.

${ }^{41}$ E. Y. Tsymbal, A. Sokolov, I. F. Sabirianov, and B. Doudin, Phys. Rev. Lett. 90, 186602 (2003). 